\title{
Venous leakage treatment revisited: Pelvic venoablation using aethoxysclerol under air block technique and Valsalva maneuver
}

\author{
Ralf Herwig ${ }^{1}$, Salvatore Sansalone ${ }^{2}$ \\ ${ }^{1}$ Vienna International Medical Clinic, Department of Urology, Austria; \\ ${ }^{2}$ Medical University Tor Vergata, Department of Urology, Italy.
}

\begin{abstract}
Summary Objective: We evaluated the effectiveness of pelvic vein embolization with aethoxysclerol in aero-block technique for the treatment of impotence due to venous leakage in men using sildenafil for intercourse. The aim of the procedure was to reduce the use of sildenafil.

Methods: A total of 96 patients with veno-occlusive dysfunction, severe enough for the need of PDE5 inhibitors for vaginal penetration, underwent pelvic venoablation with aethoxysclerol. The mean patient age was 53.5 years. Venous leaks were identified by Color Doppler Ultrasound after intracavernous alprostadil injection. Under local anesthesia a 20-gauge needle was inserted into the deep dorsal penile vein. The pelvic venogram was obtained through deep dorsal venography. Aethoxysclerol $3 \%$ as sclerosing agent was injected after air-block under Valsalva manoeuver. Success was defined as the ability to achieve vaginal insertion without the aid of any drugs, vasoactive injections, penile prosthesis, or vacuum device. Additionally, a pre- and post- therapy IIEF score and a digital overnight spontaneous erections protocol (OSEP) with the NEVA ${ }^{\mathrm{TM}}$-system was performed.

Results: At 3 month follow-up 77 out of 96 patients (80.21\%) reported to have erections sufficient for vaginal insertion without the use of any drug or additional device. Four $(4.17 \%)$ patients did not report any improvement. Follow up with color Doppler ultrasound revealed a new or persistent venous leakage in $8(8.33 \%)$ of the patients. No serious complications occurred.

Conclusions: Our new pelvic venoablation technique using aethoxysclerol in air-block technique was effective, minimally invasive, and cost-effective. All patients were able to perform sexual intercourse without the previously used dosage of PDE5 inhibitor. This new method may help in patients with contra-indications against PDE5 inhibitors, in patients who cannot afford the frequent usage of expensive oral medication or those who do not fully respond to PDE5-inhibitors.
\end{abstract}

KEY WORDS: Venous leak; Sclerotherapy; Embolization; Erectile dysfunction; Pelvic venoablation; Color Doppler ultrasound.

Submitted 2 August 2014; Accepted 30 November 2014

\section{INTRODUCTION}

PDE5-Inhibitors have become the standard therapy in erectile dysfunction (ED) over the past 20 years (1). But, most men with ED are treated with options that are not cause specific (1).

In a dose-response premarketing study, improved erections were reported after 24 weeks of treatment by $56 \%$ and $77 \%$ of men taking respectively 25 or $50 \mathrm{mg}$ of sildenafil compared with $25 \%$ of men taking placebo (1).

The efficacy of and satisfaction with sildenafil in another study in patients with venous leak were considered good for 50 patients (31.84\%), fair for 46 patients (29.29\%), and bad for 61 patients (38.85\%) (2).

In patients failing to respond to medical treatment and denying a penile implant option remain a challenge. Many of them, including those suffering ED after radical prostatectomy (RP), complain of difficulties in maintaining normally occurring erections (3). Especially younger patients complaining of the inability to maintain enough rigidity to achieve satisfactory intercourse, even after improvement by PDE5-inhibitors, are in search of a curative or at least a long term treatment (4).

Ultrasound has proven to be the major tool in the differentiation of vascular processes in erectile dysfunction $(5,6)$, particularly in patients not responding to first-line orally active drugs and seeking an explanation as to why these agents failed (6). The improvement of patient's selection by ultrasound (7) for different forms of therapy lead to a revisitation of venous leak interventions over the last years.

The guidelines for varicous veins treatment recommend a proximal compression of the veins to enhance the time the sclerosing agent is affecting the venous wall (8). This important aspect is lacking in the techniques described for sclerotherapy in penile venous leak (9-12). Therefore, we developed a technique, that allows to bring all the recommended and proven concepts of venous sclerotherapy into sclerotherapy in case of penile venous leak. This includes the use of polidocanol 3\% and injection in air-block technique under Valsalva maneuver. This procedure was performed in local anaesthesia. We report our experience with this improved technique. 


\section{Material and Methods}

Routine evaluation consisting of medical history, physical examination, complete blood analysis, hormonal analysis, biochemistry studies, IIEF15-Score and color Doppler ultrasound after intracavernous injection of $0.02 \mathrm{mg}$ alprostadil was performed in patients presenting with erectile dysfunction. Peak systolic velocity less than $25 \mathrm{~cm}$ per second was accepted as a criterion for arterial insufficiency. A resistive index (RI) lower than 0.90 in combination with an end-diastolic blood flow (EDV) of $5 \mathrm{~cm}$ or greater and persistence of flow on Valsalva maneuver according to the criterion of Fuerst and Virag was defined as venous leakage. All patients with confirmed diagnosis were informed about the results, advan-

Figure 1.

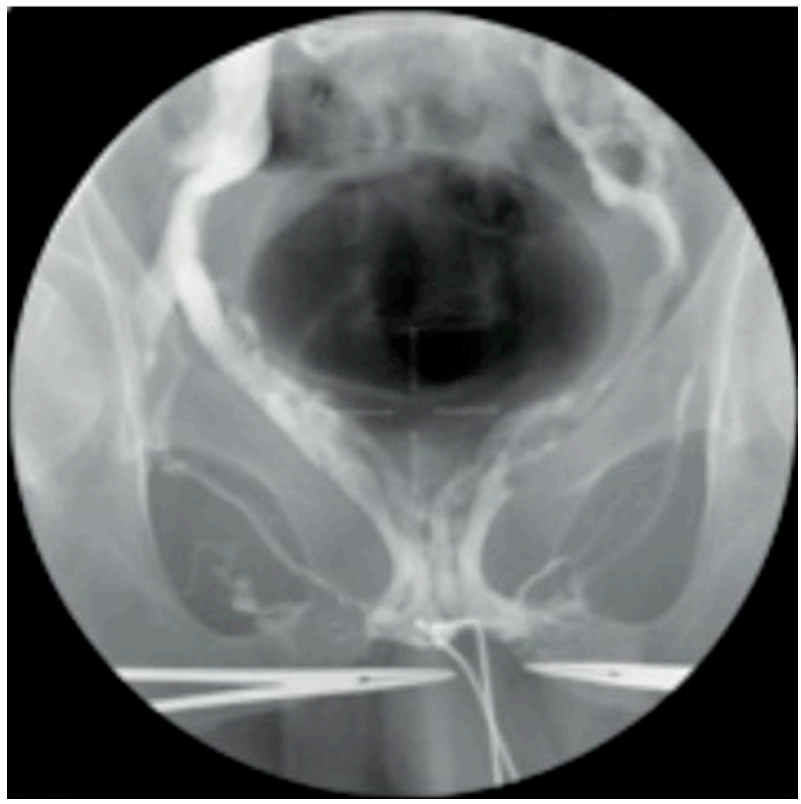

Figure 2.

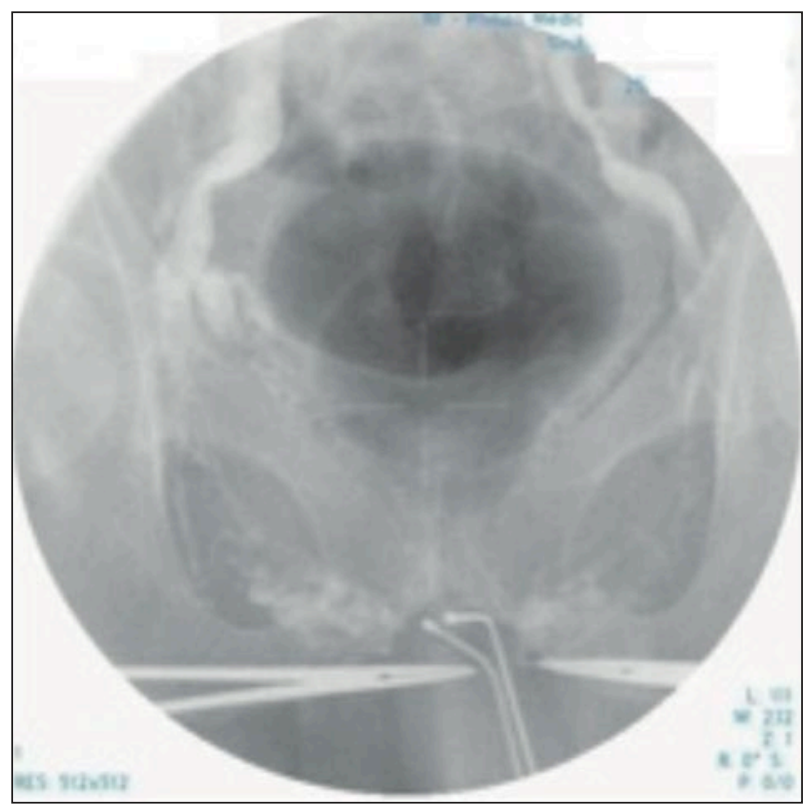

tages and disadvantages of available treatment options. Ninety-six patients diagnosed with venous leakage with a mean age of 53.5 years (range, 30 to 74) elected deep dorsal vein abllation. An overnight spontaneous erection protocol (OSEP) was obtained with the NEVATM -System (UroMetrics Inc, St. Paul, MN, USA) pre- and immediately postoperative and three months after treatment. Additionally, the IIEF15-Score was ascertained three months after therapy.

After penile block with lidocaine a 1 to $2 \mathrm{~cm}$ penile dorsal midline incision was made at the penile base. The superficial dorsal vein was ligated. The deep dorsal vein was identified and isolated for about $2 \mathrm{~cm}$ length under the Buck's fascia. The distal end was ligated with 4-0 Vicryl ${ }^{\mathrm{TM}}$ to prevent backflow into the glans penis and corpus cavernosum. Circumflexed veins, which branched off from the isolated portion of the deep dorsal vein at the penile base, were ligated with 4-0 Vicryl ${ }^{\mathrm{TM}}$, too. The proximal end was catheterized with a 20 gauge steel needle.

The venous tract was confirmed by venography (Figure 1). An air-block was set by antegrade injection of $1 \mathrm{ml}$ air followed by $4 \mathrm{ml}$ aethoxysclerol 3\% for venoablation. The patient was asked to do a Valsalva maneuver for about 3060 seconds. The occluded dorsal vein and its occluded collaterals were visualized (Figure 2). The penile incision was anatomically closed with absorbable suture material. Again an OSEP was obtained immediately after therapy. Patients were reassessed by IIEF-Score and OSEP at three months follow up.

\section{RESULTS}

The mean age at the time of surgery was 53.5 years (range 30 to 74), mean duration of disease was 3.7 years (range 0.5 to 10 ). The mean duration for the procedure was 23.8 minutes (range 12 to 41 ) with a mean use of 4 $\mathrm{ml}$ aethoxysclerol 3\% (Table 1).

At 3-months post-operative follow-up schedule 77 (80.21\%) patients reported to be able to penetrate without the aid of PDE5 inhibitors and 15 patients (15.63\%) still needed aid with PDE5 inhibitors. Four (4.17\%) patients did not report any improvement. A typical picture before and after successful sclerotherapy is shown in Figure 1. This patient presented with a doubled dorsal vein and a massive venous leak with varicous veins in the lower pelvis. These veins disappeared after sclerotherapy in the above described manner.

Follow up color Doppler ultrasound revealed a venous leakage in $8(8.33 \%)$ of the patients. Preoperative IIEF15scores changed significantly from 20.5 (range 12 to 41 ) to 72.5 (range 70 to 75$)$ at 3-months follow up ( $\mathrm{p}=0.02)$ (Table 2). Furthermore the incidence of overnight spontaneous erections increased from 2.83 preoperatively to 6.5 $(\mathrm{p}=0.002)$ at 3 -months follow up. Volumetric analysis of the penis showed a significant increase from $119.47 \%(+/-$ $74.66 \%)$ preoperatively to $258.44 \%$ (+/- $85.40 \%, \mathrm{p}=$ 0.006 ) at 3-months follows up (Table 2). After a follow up of 12 months $(\mathrm{n}=22)$ up to now $16(72.73 \%)$ patients still reported to have a strong enough erection for sexual intercourse. Four (18.18\%) patients used PDE5 inhibitors and $2(9.09 \%)$ patients did not report any change to their preoperative state. 
Table 1.

\begin{tabular}{|l|c|c|c|}
\hline & Mean & Min & Max \\
\hline Age (years) & 53.53 & 30 & 74 \\
\hline Duration of ED (years) & 3.7 & 1 & 10 \\
\hline PDE 5 inhibitor per intercourse (mg) & 75 & 50 & 100 \\
\hline Operation time (min) & 15.8 & 12 & 41 \\
\hline
\end{tabular}

Table 2.

\begin{tabular}{|l|c|c|c|}
\hline & Prae op & Post op & Significance \\
\hline IIEF 15 & 20.5 & 72.5 & 0.02 \\
\hline Spontaneous erections & 2.83 & 6.5 & 0.002 \\
\hline Volume enhancement & $119.47 \%$ & $258.44 \%$ & 0.006 \\
\hline
\end{tabular}

The procedure itself did not cause any intraoperative complication. Postoperative minor hematomas occurred in 12 patients, but resolved without any intervention.

\section{Discussion}

PDE5-inhibitors have become the first-line standard therapy in erectile dysfunction (1). Nevertheless, this therapy is not cause dependent and has a success rate at standard doses of $77 \%$ (1). This type of medication is not indicated for patients treated with certain cardiac medicaments or with other known contraindications. PDE5-inhibitors have some, not always harmless, side effects who prevent patients from continuous use. Furthermore, the medication is not covered by most health insurances and therefore is not affordable for many patients. Virag et al. reported, that after 2 years from the completion of the trial only $32 \%$ of the patients (17.2\% of the initial cohort) were still using sildenafil as a single treatment (2). In those patients who do not respond to sildenafil, a structural alteration in the components of the erectile mechanism can be suspected (1). Penile erection is believed to be the result of venous occlusion along with increased arterial inflow and corporeal smooth muscle relaxation. A failure of the closing mechanism of the penile veins is one of the most common causes of vasculogenic erectile dysfunction. In the past procedures as deep dorsal vein ligation have been performed to recover erectile function (10). However data have shown that the success rate of surgical treatments for veno-occlusive disease, especially simple penile vein ligation, is poor. The long-term data show a clear decrease of erectile ability (10). One possible explanation for this fact is the opening-up of thin collateral veins from the corpora cavernosa resulting in a similar veno-occlusive disease state as prior to the surgery at long term follow up. Our data on the contrary did not show such a significant worsening of erectile function. A possible explanation for our results may be the use of aethoxysclerol as sclerosing agent. Because of the application of the aeroblock techique in Valsalva maneouvre the sclerosing agent is maintained at place and can lead to a sufficient sclerosing of the deep dorsal vein network, including small veins. However we did experience a decrease of the success rate from about $80 \%$ at 3 months after surgery to $72 \% 12$ months after intervention. Such development may be based on the natural ability of neovascularization. Therefore it may be that in some cases sclerotherapy may lead to a temporary improvement of erectile function, which will last for a while but will ultimately result in erectile dysfunction again. Due to the minimal side effects observed, this minimal invasive method should be offered to most patients suffering from erectile dysfunction due to venous leak with either low/no effect of PDE5-inhibitor or to patients, who do not want or can not afford PDE5-inhibitor therapy.

Nevertheless in many cases this sclerosis treatment option has shown very good results, and has brought major relief for the patients in psychological as well as in psychosexual terms (9-11, 13-21).

Our technique leads to the occlusion of multiple venous channels, such as the prostatic plexus, internal pudendal veins and inferior vesical veins. Aethoxysclerol is a sclerosing agent that has been used extensively in varicositas treatment. To our knowledge no study has reported the use of aethoxysclerol for the treatment of venogenic erectile dysfunction. An injection of this agent into a vein leads to a permanent emobolization and consequent closure of the vein. This features have been used for the treatment of varicose veins for many decades (12) (22). Although we have not observed any event like this, it exists the possibility of small migrations in the lungs and before the procedure it should be diagnosed the presence of an open foramen oval in between the two auricles. Use of the principles of well known procedures (12) in combination with current findings in anatomy $(4,23)$ may lead to better long-term result in venous leak patients. May be that this could lead to a minimal invasive procedure, that offers more options than penile prosthesis after PDE5-inhibitor failure or in patients who cannot use this therapeutic option. Our short-term and preliminary intermediate term results are encouraging and should be followed-up in the future.

\section{Conclusions}

Our new pelvic venoablation technique using aethoxysclerol in air-block technique was effective, minimally invasive, and cost-effective. All patients were able to perform sexual intercourse without the previously used dosage of their PDE5 inhibitor. This new method may help in patients with contra-indications against PDE5 inhibitors, in patients who can not afford the frequent usage of expensive oral medications or those who do not fully respond to PDE5-inhibitors.

\section{References}

1. Hatzimouratidis K, Amar E, Eardley I, et al. Guidelines on male sexual dysfunction: erectile dysfunction and premature ejaculation. Eur Urol. 2010; 57:804-814.

2. Virag R Indications and early results of sildenafil (Viagra) in erectile dysfunction. Urology. 1999; 54:1073-1077.

3. Nehra A, Goldstein I, Pabby A, et al. Mechanisms of venous leakage: a prospective clinicopathological correlation of corporeal function and structure. J Urol. 1996; 156:1320-9. 
4. Virag R, Paul JF. New classification of anomalous venous drainage using caverno-computed tomography in men with erectile dysfunction. J Sex Med. 2011; 8:1439-1444.

5. Altinkilic B, Hauck EW, Weidner W Evaluation of penile perfusion by color-coded duplex sonography in the management of erectile dysfunction. World J Urol. 2004; 22:361-4.

6. Aversa A, Sarteschi LM The role of penile color-duplex ultrasound for the evaluation of erectile dysfunction. J Sex Med. 2007; 4:14371447.

7. Virag R, Sussman H. Exploration of the deep dorsal vein of the penis using pulsed Doppler ultrasonography. Preliminary study. J Mal Vasc. 1998; 23:195-198.

8. Campbell B. Varicose veins and their management. BMJ. 2006; 333:287-92

9. Henriet JP Value of sclerotherapy in the treatment of certain types of impotence caused by venous leakage. Phlébologie. 1987; 40:975.

10. Henriet, JP. Value of sclerotherapy in the treatment of certain types of impotence caused by venous leakage. Phlebologie. 1987; 40:975-980.

11. Miwa Y, Shioyama R, Itou $Y$, et al. Pelvic venoablation with ethanol for the treatment of erectile dysfunction due to veno-occlusive dysfunction. Urology. 2001; 58:76-9.

12. Nakata M, Takashima S, Kaminou T, et al.. Embolotherapy for venous impotence: use of ethanol. J Vasc Interv Radiol. 2000; 11:1053-1057.

13. Redondo P, Cabrera J. Microfoam sclerotherapy. Semin Cutan Med Surg. 2005; 24:175-83.

14. Basche S1, Eger C, Elsebach K, Ulshöfer B. Veno-occlusive dysfunction as a cause of erectile impotence: therapy of venous leak with retrograde embolization of the internal pudendal vein. Vasa. 2003; 32:47-50.
15. Herwig R, Margreiter M, Kuehhas F. Pelvic venoablation for the treatment of erectile dysfunction caused by venous leakage. Eur Urol Suppl. 2012; 11:87

16. Kutlu R, Soylu A. Deep dorsal vein embolization with N-butyl2-cyanoacrylate and lipiodol mixture in venogenic erectile dysfunction: early and late results. Radiol Oncol. 2009; 43:17-25.

17. Muller SC, Schild H, Fritz T, Witzsch U. Percutaneous transpenile and retrograde venous occlusion for the treatment of venous leak impotence. Eur Urol. 1991; 19:101-103.

18. Peskircioglu L, Tekin I, Boyvat F, et al. Embolization of the deep dorsal vein for the treatment of erectile impotence due to veno-occlusive dysfunction. J Urol. 2000; 163:472-5.

19. Popken G, Katzenwadel A, Wetterauer U. Long-term results of dorsal penile vein ligation for symptomatic treatment of erectile dysfunction. Andrologia. 1999; 31: 77-82.

20. Schild H, Müller SC, Bürger RA, Fritz T. Transpenile venous occlusion in the treatment of erectile impotence.Rofo. 1989; 151:470-2.

21. Vale JA, Feneley MR, Lees WR, Kirby RS. Venous leak surgery: long-term follow-up of patients undergoing excision and ligation of the deep dorsal vein of the penis. Br J Urol. 1995; 76:192-195.

22. Zhang B, Chen J, Xiao H, et al. Treatment of penile deep dorsal venous leakage of erectile dysfunction by embedding the deep dorsal vein of the penis: a single center experience with 17 patients. J Sex Med. 2009; 6:1467-1473.

23. Hsu GL, Chen HS, Hsieh CH, et al. Insufficient response to venous stripping surgery: is the penile vein recurrent or residual? J Androl. 2006; 27:700-706.

24. Paul JF, Virag R. Does anatomy of the pubic arch interfere with the maintaining of erection? J Sex Med 2013; 10:777-781.

\section{Correspondence}

Ralf Herwig, MD, Professor (Corresponding Author)

dr.ralf.herwig@gmail.com

Vienna International Medical Clinic

Kreuzgasse 17-19 - 1170 Vienna (Austria)

Salvatore Sansalone, MD

salvatore.sansalone@yahoo.it

Viale Oxford, 81 - 00133 Rome, Italy 